\title{
Nausea During Pregnancy and Timing of Pubertal Development in Sons and Daughters: A Population-Based Cohort Study
}

\author{
Mette Rahbek Bruun' \\ Andreas Ernst ${ }^{1,2}$ \\ Linn Håkonsen Arendt ${ }^{1,3}$ \\ Cecilia Høst Ramlau- \\ Hansen (D) ' \\ Nis Brix ${ }^{1,4}$ \\ 'Department of Public Health, Research \\ Unit for Epidemiology, Aarhus University, \\ Aarhus, Denmark; ${ }^{2}$ Department of \\ Urology, Aarhus University Hospital, \\ Aarhus, Denmark; ${ }^{3}$ Department of \\ Obstetrics and Gynecology, Regional \\ Hospital Horsens, Horsens, Denmark; \\ ${ }^{4}$ Department of Clinical Genetics, Aarhus \\ University Hospital, Aarhus, Denmark
}

Purpose: The objective of this study was to explore whether maternal nausea in pregnancy, a potential surrogate marker of endogenous estrogen levels, was associated with age at attaining pubertal milestones in sons and daughters.

Materials and Methods: The study included a total of 14,612 boys and girls born in 20002003, from the Danish National Birth Cohort. Information on nausea was reported by mothers during pregnancy in telephone interviews scheduled around gestational week 12 and 30. Their children were invited every six months from 11 years of age until 18 years of full maturation to provide information on current pubertal status. Pubertal milestones included pubic hair, axillary hair and acne for both sexes, besides genital development, voice break and first ejaculation for boys, and breast development and menarche for girls. Mean monthly differences in age at attaining several pubertal milestones for boys and girls were estimated according to duration of nausea in the first trimester $(0,1-6,7-11,12$ weeks). Further, we explored whether duration of nausea in the first two trimesters $(0,1-8,9-15$ or 16-28 weeks) and severity (measured by co-existence of vomiting and weight loss) were associated with pubertal timing.

Results: Neither maternal nausea in the first trimester nor duration or severity of nausea in the first two trimesters were associated with pubertal timing.

Conclusion: Our study does not support the hypothesis that nausea in pregnancy - as a surrogate marker of endogenous estrogen levels - is associated with age at attaining pubertal milestones in children.

Keywords: maternal exposure, prenatal exposure delayed effects, nausea, puberty, estrogen

\section{Plain Language Summary}

The aim of this study was to examine whether children born by mothers with nausea in pregnancy experience earlier pubertal timing compared to children born by mothers without nausea. Studies have also shown that children with earlier age at pubertal milestones have a higher risk of developing diseases in adulthood, including obesity, cardiovascular disease, diabetes mellitus and cancers. Therefore, we have great interest in identifying potential causes of earlier onset of puberty. Based on earlier literature, nausea in pregnancy may be linked to higher levels of estrogen in pregnant women, and thereby higher fetal levels of estrogen exposure which has been suggested to accelerate pubertal development in the offspring. In this study of 14,612 Danish children born in 2000-2003, we studied whether the duration and severity of nausea (measured by co-existence of vomiting and weight loss) in early pregnancy may affect age at attaining specific pubertal milestones. Information on nausea during pregnancy including duration and severity was collected through telephone-assisted computer
Correspondence: Mette Rahbek Bruun Email mettebruun_90@hotmail.com (c) (7) (ㄱ) 2021 Bruun et al. This work is published and licensed by Dove Medical Press Limited. The full terms of this license are available at https://www.dovepress.com/terms. (c) $\mathrm{BY}_{\mathrm{NC}}$ php and incorporate the Creative Commons Attribution - Non Commercial (unported, v3.0) License (http://creativecommons.org/licenses/by-n//3.0/). By accessing the work you hereby accept the Terms. Non-commercial uses of the work are permitted without any further permission from Dove Medical Press Limited, provided the work is properly attributed. For permission for commercial use of this work, please see paragraphs 4.2 and 5 of our Terms (https://www.dovepress.com/terms.php). 
interviews with the mothers twice during their pregnancy. Information on the pubertal stages of the children was collected through web-based questionnaires from the children starting at the age of 11 and every six months throughout puberty. We did not find an association between pregnant women experiencing nausea and earlier age at pubertal milestones in their children.

\section{Introduction}

A time trend toward earlier age at pubertal development has been observed in girls in Western countries, but a time trend is less certain in boys. ${ }^{1-4}$ Earlier timing of puberty has been associated with several diseases in the adulthood including obesity, type 2 diabetes, cardiovascular diseases, testicular cancer and breast cancer. ${ }^{1,5}$ Thus, it is of public health importance to investigate potential causes of earlier onset of puberty.

The initiation and progression of puberty are under control of the hypothalamic-pituitary-gonadal axis (HPG axis) and adrenal androgens, and these systems are founded and matured during prenatal life. ${ }^{6,7}$ Early prenatal exposure to higher level of endogenous estrogens may interfere with the programming of these early developmental processes, potentially altering the timing of pubertal development through changes of the negative feedback responses. ${ }^{6}$

Nausea during pregnancy may be associated with earlier age at pubertal timing since nausea during pregnancy may be a possible surrogate marker of fetal exposure to endogenous estrogens. A relatively higher level of estrogen is possibly present in pregnant women with nausea and vomiting during pregnancy. ${ }^{8}$ Women with severe nausea and vomiting during pregnancy may have higher levels of pregnancy-related hormones, including human choriogonadotropin (hCG), ${ }^{9-13}$ which has been observed in studies on twin pregnancies, hyperemesis gravidarum and mole pregnancy. These high levels of pregnancy-related hormones stimulate the corpus luteum to produce and release estrogen and progesterone in early pregnancy, which may result in a higher fetal exposure. ${ }^{14}$ As endocrine disrupting chemicals with agonistic activity on the estrogen receptor have been suggested to alter pubertal timing, ${ }^{15-17}$ exposure to endogenous estrogen would plausibly have a similar effect. Furthermore, studies have suggested slower intestinal transit time and gastric emptying as a result of higher levels of estrogen during pregnancy potentially leading to hyperemesis gravidarum. ${ }^{9,10,18}$ This supports the use of nausea and vomiting during pregnancy as a marker of higher estrogen levels.
Both prevention and treatment of nausea and vomiting during pregnancy have been extensively studied, and recent guidelines present both non-pharmacological and pharmacological strategies. ${ }^{19}$ Non-pharmacological prevention strategies for the pregnant woman with low to moderate severity of symptoms include frequent small meals, treatment with ginger, acupressure and psychotherapy, as well as avoiding iron supplements, spicy and fatty foods. Further, taking multivitamin prior to conception has been suggested as another possible strategy to reduce symptoms. ${ }^{19}$ More severe degrees of nausea and vomiting during pregnancy may be treated with antihistamines, phenothiazines, dopamine and serotonin 5-hydroxytryptamine type 3 receptor antagonist. $^{20-22}$

In Denmark, all pregnant women are invited to follow a standardized follow-up program combined of routine consultations with their general practitioner, midwives and at public hospitals where ultrasound scans are performed among others. The birth of the baby is always managed by public hospitals. If nausea and vomiting are too severe and cannot be managed at the general practitioner or pharmacological treatment is necessary, the pregnant women is admitted to a public hospital.

Several animal studies on sheep and baboons have found earlier timing of puberty after prenatal exogenous estrogen exposure, ${ }^{16,23}$ whereas results from rodents have been ambiguous. ${ }^{15,24}$ Human studies have investigated potential effects on timing of pubertal development after prenatal endogenous estrogen exposure, based on direct measurements of estrogen levels, as well as exogenous estrogenic exposures such as diethylstilbestrol, phthalates and phytoestrogens. ${ }^{11,17}$ These studies have not consistently shown an association between prenatal exposure to estrogens and changes of pubertal development.

We hypothesized that nausea during pregnancy, considered a potential surrogate marker of endogenous estrogen exposure, is associated with earlier timing of pubertal development in the children. The aim of this study was, therefore, to examine whether boys and girls born by mothers with nausea during pregnancy achieved various pubertal markers earlier than children born by mothers without nausea during pregnancy from a large longitudinal Danish puberty cohort.

\section{Materials and Methods}

\section{Study Population}

This population-based cohort study is based on data from the Puberty Cohort, a sub-cohort in the Danish National Birth 
Cohort (DNBC) ${ }^{25}$ The DNBC holds data on approximately 92,000 mothers and their more than 100,000 children born in the period from 1996 to 2003. Information on maternal lifestyle, pregnancy and birth outcomes has been collected through two computer-assisted telephone interviews scheduled around gestational week 12 and 30 , as well as six and 18 months after the pregnancy. Furthermore, follow-up questionnaires were mailed or emailed to the mothers when the children reached seven and 11 years of age. Information on maternal characteristics was shown in Table 1.

In May 2012, 22,439 of 56,641 children were invited for participation in the Puberty Cohort (Figure 1). Children eligible for being sampled to the Puberty Cohort were liveborn singletons, born between 2000 and 2003, whose mothers had participated in the first interview in DNBC without withdrawal of their informed consent $(n=56,641)$.
To increase the statistical efficiency, children were sampled from 12 prenatal exposure groups, hypothesized to be potential causes of earlier pubertal timing, and a random sample of 8000 children. ${ }^{26}$ Since August 2012, web-based questionnaires have been used to obtain self-reported, longitudinal information on pubertal development collected every six months from 11.5 years of age until 18 years or full maturity. Full maturity was defined as having attained both Tanner stages 5 for pubic hair and genital development in boys and Tanner stage 5 for pubic hair and breast development in girls. Finally, identical information on puberty was provided as part of the 11-year follow-up of the entire DNBC.

\section{Nausea, Vomiting and Weight Loss During Pregnancy}

Information on nausea, vomiting and weight loss during pregnancy was extracted from the baseline interviews

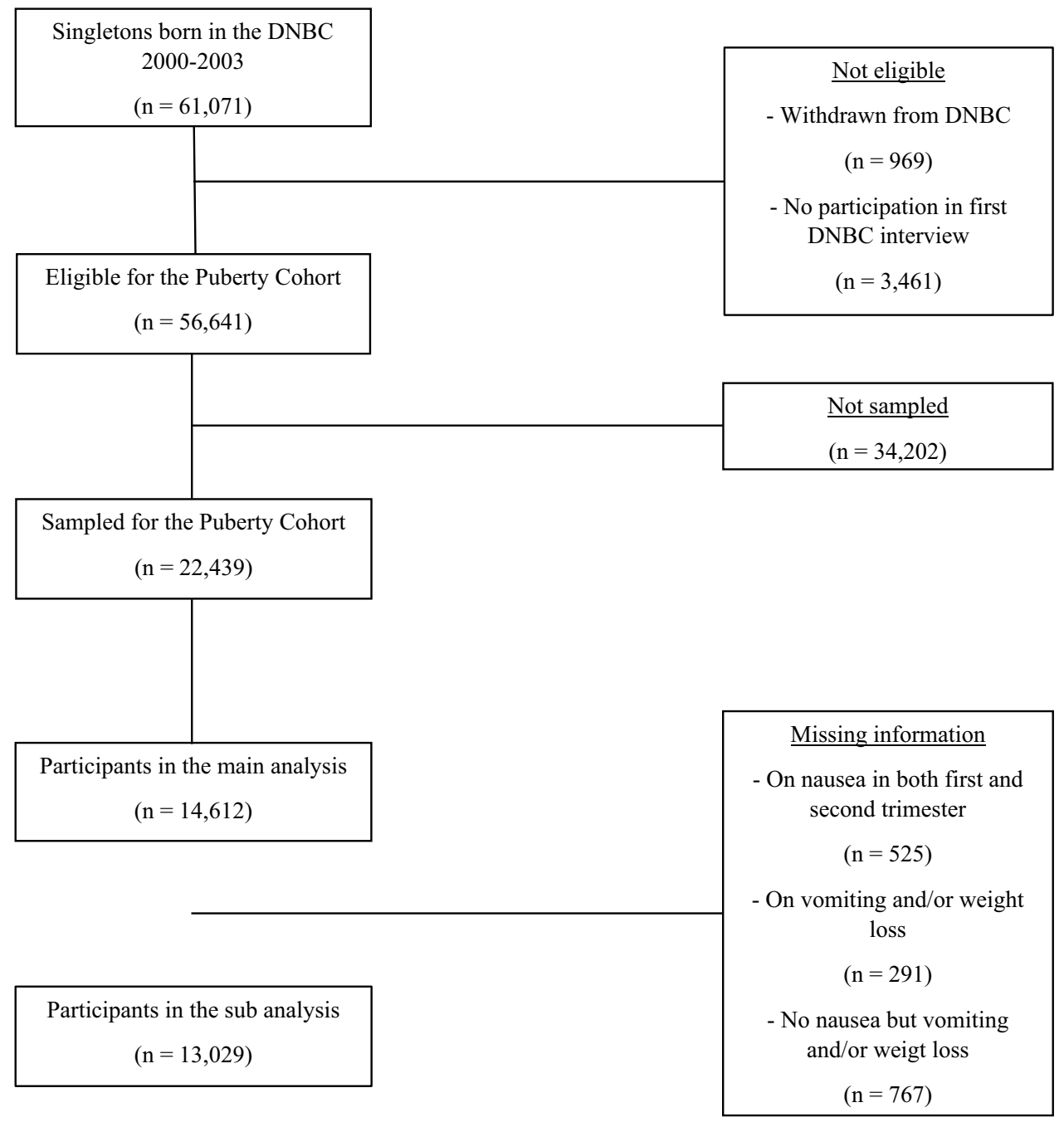

Figure I Flowchart of participants in the Puberty Cohort, Denmark, 2012-20I8. 
Table I Background Characteristics According to Nausea in the First Trimester for Children in the Puberty Cohort $(n=14,612)$, Denmark

\begin{tabular}{|c|c|c|c|c|c|}
\hline & & $\begin{array}{l}\text { Nausea in First } \\
\text { Trimester }\end{array}$ & & & \\
\hline & 0 Weeks & I-6 Weeks & $\begin{array}{c}\text { 7-II } \\
\text { Weeks }\end{array}$ & All Weeks & $\begin{array}{l}\text { Missing } \\
\text { (\%) }\end{array}$ \\
\hline n (\%) & $6380(43.7)$ & $3139(21.5)$ & $4385(30.0)$ & $708(4.8)$ & $1207(7.63)$ \\
\hline \multicolumn{6}{|l|}{ Maternal characteristics } \\
\hline \multicolumn{6}{|l|}{$\begin{array}{l}\text { Daily number of cigarettes in first trimester, } \\
\text { n (\%) }\end{array}$} \\
\hline Non-smoker & $4226(66.4)$ & $2372(75.7)$ & $3407(77.9)$ & $537(75.8)$ & \\
\hline $0-10$ cigarettes/day & $1676(26.3)$ & $610(19.5)$ & $788(18.0)$ & $135(19.1)$ & \\
\hline 10 cigarettes/day & $462(7.3)$ & $|5|(4.8)$ & $178(4.1)$ & $36(5.1)$ & $53(0.34)$ \\
\hline \multicolumn{6}{|l|}{ Pre-pregnancy BMI, n (\%) } \\
\hline$<18.5$ & $392(6.2)$ & $213(6.9)$ & $314(7.2)$ & $35(5.0)$ & \\
\hline $18.5-24.9$ & $3997(63.5)$ & $1932(62.5)$ & $2578(59.5)$ & $414(59.6)$ & \\
\hline $25.0-29.9$ & $1299(20.6)$ & $635(20.5)$ & $977(22.5)$ & $158(22.7)$ & \\
\hline$>30.0$ & $605(9.6)$ & $3 I I(10.1)$ & $467(10.8)$ & $88(12.7)$ & $217(1.37)$ \\
\hline \multicolumn{6}{|l|}{ Maternal age of menarche, $n(\%)$} \\
\hline Earlier than peers & I547 (24.4) & $799(25.7)$ & || $4 \mid(26.2)$ & $205(29.3)$ & \\
\hline Same time as peers & $3687(58.2)$ & $1766(56.9)$ & $2491(57.2)$ & $359(5 I .3)$ & \\
\hline Later than peers & $1106(17.4)$ & $54 \mid(17.4)$ & $720(16.5)$ & $136(19.4)$ & $123(0.78)$ \\
\hline Maternal age at delivery in years, mean (SD) & $30.5(4.4)$ & $30.4(4.3)$ & $30.9(4.3)$ & $30.8(4.7)$ & $6(0.04)$ \\
\hline \multicolumn{6}{|l|}{ Parity, n (\%) } \\
\hline First child & $3676(57.6)$ & $1534(48.9)$ & I77I (40.4) & $334(47.2)$ & \\
\hline Second or more child & $2704(42.4)$ & $1605(51.1)$ & $2614(59.6)$ & $374(52.8)$ & $0(0.00)$ \\
\hline \multicolumn{6}{|l|}{ Highest social class of parents, $\mathbf{n}(\%)$} \\
\hline High grade professional & $1497(23.5)$ & $754(24.1)$ & $1016(23.2)$ & $152(2 \mid .5)$ & \\
\hline Low grade professional & $2003(31.4)$ & $1017(32.5)$ & $|53|(35.0)$ & $244(34.5)$ & \\
\hline Skilled worker & I828 (28.7) & $838(26.8)$ & $1169(26.7)$ & $193(27.3)$ & \\
\hline Unskilled worker & $883(13.9)$ & $432(13.8)$ & $56 \mid(12.8)$ & $99(14.0)$ & \\
\hline Student & $|3|(2.1)$ & $76(2.4)$ & $70(1.6)$ & $12(1.7)$ & \\
\hline Economically inactive & $30(0.5)$ & $15(0.5)$ & $27(0.6)$ & $7(1.0)$ & $31(0.20)$ \\
\hline Birth weight, mean (SD) & $35 \mid 4(567.2)$ & $3554(552.7)$ & $3614(550.8)$ & $3533(563.1)$ & $57(0.36)$ \\
\hline Childhood BMI, mean (SD) & I5.7 (I.7) & $15.6(1.7)$ & I5.7 (I.7) & I5.7 (I.8) & $4773(30.2)$ \\
\hline
\end{tabular}

conducted at approximately 12 and 30 weeks of gestation. The mothers were asked whether they had experienced nausea, vomiting or weight loss. If so, they subsequently provided information on the specific gestational weeks with nausea, vomiting and weight loss. From these data, we derived the main exposure; "nausea in first trimester" ( 0 weeks, 1-6 weeks, 7-11 weeks or 12 weeks). Nausea in first trimester was considered the main exposure as the HPG axis develops and matures during this early prenatal period. $^{7}$
For a sub-analysis, we derived a two-dimensional exposure variable intended to capture both duration and severity of nausea. First, we derived "duration of nausea in first two trimesters" (0 weeks, 1-8 weeks, 9-15 weeks or 16-28 weeks). Secondly, we derived "severity of nausea" by combining the information on nausea, vomiting and weight loss (no nausea, nausea only, nausea and vomiting, nausea and weight loss, or nausea, vomiting and weight loss). We combined the two variables to obtain the new exposure categorization with 12 different exposure levels. The sub-analysis with 12 
exposure levels based on the duration and severity of nausea (measured by co-existence with vomiting and weight loss) were considered exploratory investigating potential doseresponse patterns with pubertal development. If in fact nausea and vomiting are a surrogate marker of endogenous estrogen exposure, the duration and severity of symptoms could represent the level (or dose) of exposure.

\section{Timing of Puberty}

Information on current stage of various pubertal milestones was collected by the half-yearly questionnaires: for both sexes, axillary hair (yes, no) and acne (yes, no); for boys, pubic hair (Tanner stages, PH1-5), genital development (Tanner stages, G1-5), voice break (yes sometimes, yes definitively, no or do not know) and first ejaculation (yes, no; if yes: year and month); and for girls, pubic hair (Tanner stages, PH1-5), breast development (Tanner stages, B1-5) and menarche (yes, no; if yes: year and month). The questionnaires included illustrations of Tanner stages 1-5 and a description of each stage. $^{25}$

\section{Covariates}

Using a directed acyclic graph, the following covariates were identified as possible confounding factors in this study: maternal pre-pregnancy body mass index (BMI), maternal parity, maternal age at delivery, maternal age at menarche, smoking status and highest educational class of parents. The covariates were categorized as shown in Table 2 and adjusted for in the analyses. Information on parity and maternal age at delivery was obtained from the Danish Medical Birth Register. ${ }^{27}$ Information on highest educational class of parents was retrieved from the DNBC and was based on the International Standard Class of Occupation and Education codes (ISCO-88 and ISCED). ${ }^{28}$ Information on the remaining potential confounders was obtained from the DNBC provided by the women during pregnancy. For sub-analyses, we used information on birth weight obtained from the Danish Medical Birth Register, and information on BMI of the children at seven years of age obtained from the sevenyear follow-up in the DNBC.

\section{Statistical Analysis}

We considered values outside the following ranges as outliers and recoded them as missing; childhood BMI at seven years was restricted to those with height $>100$ and $<150 \mathrm{~cm}$ as well as weight $<90 \mathrm{~kg}$ (childhood BMI for eight children was recoded as missing); birth weight was restricted to those between $\geq 400$ and $<6000$ (birth weight for 47 children was recoded as missing); and maternal age were restricted to those between 15 and 50 years (none were recoded as missing).

In the primary analysis, we used a multivariable regression model for censored data fitted by maximum likelihood estimation using STATA's -intreg- procedure. This approach assumes normally distributed residuals. In the main analysis, we estimated the adjusted mean monthly difference of age at attaining the different pubertal milestones for gestational weeks with nausea in the first trimester using no nausea as the reference. Categorical confounding variables were introduced in the regression model as indicator variables, and maternal age at birth (continuous variable) was introduced as a second order polynomial. We assessed the assumption of normally distributed residuals by plotting the nonparametric cumulative incidence function of the residuals based on the Turnbull estimator against the parametric cumulative incidence function based on the normal distribution. ${ }^{29}$ The plots were then stratified by levels of covariates to assess the assumption of constant variance. The plots were compatible with both assumptions (data not shown).

Our variable capturing both duration and severity of nausea was used as the exposure of interest in a subanalysis. We obtained the mean monthly difference in timing of puberty by modelling all pubertal milestones simultaneously using Huber-White robust variance estimation to obtain a single estimate for timing of puberty in each sex across the 12 exposure categories. ${ }^{30,31}$ In two additional sub-analyses, we further adjusted the main analysis for birth weight and adjusted results for BMI of the offspring at seven years of age, respectively, to explore potential mediation by these factors.

Based on rules from probability theory, exact sampling probabilities were derived from the sampling fraction for each pre-defined sampling frame and the random sample. The inverse of these probabilities was used as sampling weights to reweight the sample to represent all children eligible for being sampled to the Puberty Cohort. The sampling strategy and derivation of sampling weight have been described in detail previously. ${ }^{26}$ To account for nonparticipation in the Puberty Cohort, selection weights were employed. $^{32}$ Selection weights were estimated by multivariable logistic regression to obtain the inverse probability of participation conditional on the exposure and the 
confounders listed above. Then, the selection weights were multiplied with the sampling weights to obtain the final weights applied in all analyses. Robust standard errors were used to account for clustering of siblings $(n=348)$ and the use of weights.

All confidence intervals were based on two-tailed tests with alpha levels of 0.05 . All analyses were conducted in STATA 15.1 MP software (StataCorp LP, College Station, TX, USA) and R (x64 3.3.1).

\section{Results}

In total, 15,819 of the 22,439 invited children participated $(70 \%)$ by returning at least one questionnaire on pubertal timing. Information on nausea in first trimester was available for 14,612 of the 15,819 children with information on pubertal development (Figure 1).

A total of $56 \%$ of the women reported nausea at any time in the first trimester, $23 \%$ reported vomiting and $16 \%$ experienced weight loss at any time in the first trimester. Mothers reporting nausea in pregnancy were more often non-smokers, had a slightly higher pre-pregnancy BMI and were of higher parity than mothers not reporting nausea (Table 1).

\section{Primary Analysis}

Nausea in the first trimester was not associated with age at achieving the pubertal milestones in sons and daughters (Figure 2A and B).

\section{Sub-Analysis}

In a sub-analysis, we explored whether duration of nausea and severity of nausea were associated with pubertal timing (Table 2). When combining all estimates for nausea only, mean monthly age differences for sons in the first two trimesters were $-0.4(-1.4,0.7)$ for $1-8$ weeks, -0.4 $(-1.6,0.8)$ for $8-15$ weeks and $-0.9(-3.3,1.5)$ for $>16$ weeks (Table 2). Likewise, mean monthly differences for daughters when combining all estimates for nausea only in the first two trimesters were $0.8(-0.2,1.8)$ for $1-8$ weeks, $0.6(-0.7,1.8)$ for $8-15$ weeks and $-1.8(-4.4,0.9)$ for $>16$ weeks (Table 2). The point estimates indicated slightly earlier age of attaining the pubertal milestones among sons across most categories of severity and duration compared to the reference group (Table 2), but the estimates were relatively small, and $95 \%$ confidence intervals overlapped the null. No dose-dependency was observed. For daughters, no consistent pattern was observed (Table 2). Further, in the last two sub-analyses with adjustment for birth weight and childhood BMI, respectively, the results remained essentially unchanged (data not shown).

\section{Discussion}

This study did not support the hypothesis that nausea, as a marker of endogenous estrogen exposure during pregnancy, is associated with earlier age at pubertal timing possibly by interfering with the foundation and maturation of the HPG axis responsible for future pubertal

Table 2 Mean Age Difference for Overall Pubertal Timing, The Puberty Cohort $(n=|4,6| 2)$, Denmark, 20I2-20I8

\begin{tabular}{|c|c|c|c|c|c|c|c|c|}
\hline \multirow[t]{3}{*}{ Severity of Nausea } & \multicolumn{8}{|c|}{ Weeks of Nausea in First two Trimesters } \\
\hline & \multicolumn{2}{|c|}{ No Nausea } & \multicolumn{2}{|c|}{ I-8 Weeks } & \multicolumn{2}{|c|}{ 8-15 Weeks } & \multicolumn{2}{|c|}{$>16$ Weeks } \\
\hline & No. & & No. & Adj. Estimate & No. & Adj. Estimate & No. & Estimate \\
\hline Sons & & & & & & & & \\
\hline Nausea, vomiting and weight loss & & & 135 & $-0.2(-2.7,2.3)$ & 230 & $\mathrm{I} .5(-0.3,3.4)$ & 126 & $0.6(-1.9,3.2)$ \\
\hline Nausea and weight loss & & & 190 & $0.6(-1.6,2.7)$ & $|3|$ & $-0.3(-2.4,1.8)$ & 42 & $-3.2(-7.4,1.1)$ \\
\hline Nausea and vomiting & & & 341 & $-0.4(-1.9,1.1)$ & 362 & $-0.6(-2.2,1.0)$ & 170 & $0.7(-1.6,2.9)$ \\
\hline Nausea & & & 1025 & $-0.4(-1.4,0.7)$ & 662 & $-0.4(-1.6,0.8)$ & 135 & $-0.9(-3.3,1.5)$ \\
\hline No nausea & 2444 & Ref. & & & & & & \\
\hline Daughters & & & & & & & & \\
\hline Nausea, vomiting and weight loss & & & 172 & $0.0(-1.9,1.8)$ & 290 & $-0.7(-2.5,1.0)$ & 190 & $-1.7(-4.0,0.6)$ \\
\hline Nausea and weight loss & & & 174 & $0.5(-1.6,2.6)$ & 178 & $-0.3(-2.2,1.6)$ & 29 & $-4.2(-10.5,2.1)$ \\
\hline Nausea and vomiting & & & 450 & $0.4(-1.0,1.9)$ & 468 & $0.0(-1.5,1.5)$ & 200 & $0.4(-1.3,2.2)$ \\
\hline Nausea & & & 1154 & $0.8(-0.2,1.8)$ & 696 & $0.6(-0.7,1.8)$ & 139 & $-1.8(-4.4,0.9)$ \\
\hline No nausea & 2413 & Ref. & & & & & & \\
\hline
\end{tabular}

Note: a Mean monthly difference in age at attaining all pubertal milestones using robust variance estimation. 
A

Tanner G3
Tanner G4
Tanner G5
Tanner PH2
Tanner PH3
Tanner PH4
Tanner PH5
Axillary hair
Acne
Voice break

First ejaculation

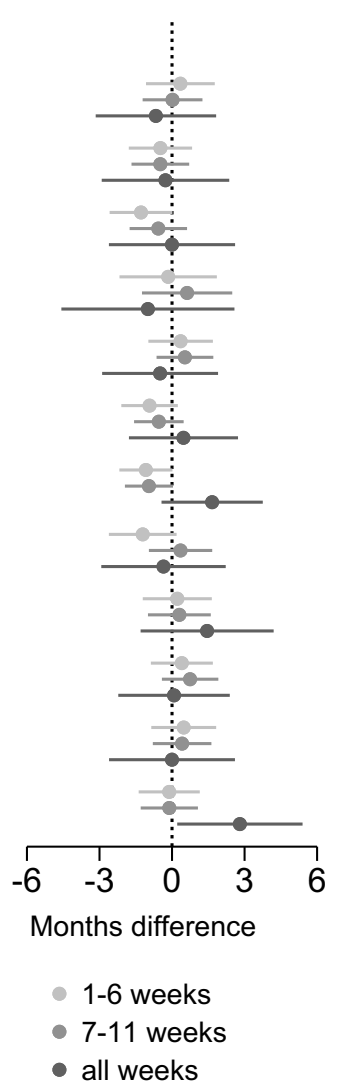

B

Tanner B2
Tanner B3
Tanner B4
Tanner B5
Tanner PH2
Tanner PH3
Tanner PH4
Tanner PH5
Axillary hair
Acne
Menarche

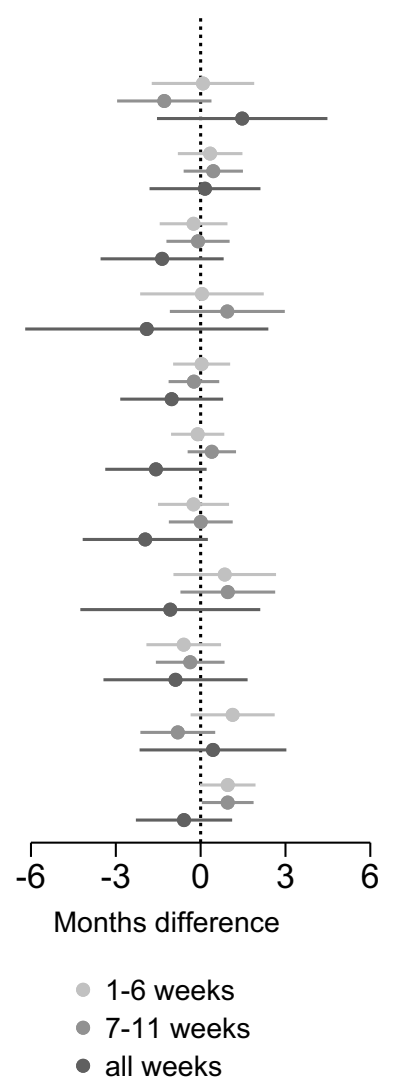

Figure 2 (A) Age difference in timing of puberty among sons in relation to maternal nausea in first trimester, the Puberty Cohort, Denmark. Estimated age difference in age at attaining pubertal milestones with $95 \%$ confidence interval with women not experiencing nausea during pregnancy as the reference and adjusted for maternal prepregnancy body mass index, maternal parity, maternal age at delivery, maternal menarche age, smoking status and highest educational class of parents. (B) Age difference in timing of puberty among daughters in relation to maternal nausea in first trimester, the Puberty Cohort, Denmark. Estimated age difference in age at attaining pubertal milestones with $95 \%$ confidence interval with women not experiencing nausea during pregnancy as the reference and adjusted for maternal pre-pregnancy body mass index, maternal parity, maternal age at delivery, maternal menarche age, smoking status and highest educational class of parents.

Abbreviations: Tanner B2-5, Tanner Breast stage 2-5; Tanner PH2-5, Tanner Pubic Hair stage 2-5.

development. To the best of our knowledge, this is the first study on the subject.

The natural course of nausea in pregnancy resembles that of hCG, and hCG stimulates the synthesis of estrogen and progesterone in the corpus luteum. After the first trimester, the estrogen level peaks, whereas the level of hCG diminishes as placental hormones take over the stimulation of maternal estrogen production. ${ }^{18}$ Considering both the close relation between estrogen and hCG during pregnancy and the similar course of maternal nausea and level of hCG, we hypothesized that maternal nausea is a marker of higher prenatal estrogen exposure, which may lead to earlier pubertal development in sons and daughters. ${ }^{6}$ However, we cannot exclude that other mechanisms might be at play; nausea might also be caused by maternal psychosocial comorbidities, weight loss during pregnancy and micronutrient deficiency, ${ }^{33,34}$ and gastrointestinal changes for protection of the mother and fetus from toxic substances. ${ }^{18}$ Furthermore, we cannot rule out that nausea in pregnancy may be a too weak marker of in utero estrogen levels, which could potentially explain the null findings of the present study.

Nausea and vomiting during pregnancy have, besides a suggested long-term risk of earlier age at timing of puberty in the children, also other possible complications. Nausea and vomiting during pregnancy have been associated with complications to the infant which is in risk of being small for gestational age, having a low birth weight or being born preterm. Furthermore, it has been suggested that severe maternal nausea and vomiting during pregnancy is associated with higher childhood total body fat mass, increased risk of cardiovascular disease and hypertension in adulthood due to extreme maternal undernutrition. ${ }^{35-37}$ Thus, identifying, preventing and 
treating nausea and vomiting in early pregnancy may have positive health implications.

This study had strengths and limitations that need to be considered. Important strengths were the large populationbased sample with longitudinal collection of information on puberty, detailed information on potential confounding factors (in particular maternal age at menarche), and the detailed information on maternal nausea, which gave us the opportunity to graduate nausea into categories based on both duration and severity to assess for dose-response patterns. Furthermore, the sub-analyses included data from the first two trimesters with information on vomiting and weight loss to isolate the most highly exposed women (in example those with nausea, vomiting and weight loss and those with nausea beyond the first trimester). Low birth weights and childhood overweight have been associated with earlier age at pubertal timing in girls. ${ }^{38}$ In the sub-analyses, we considered both birth weight and BMI of the children as potential intermediary factors, since intrauterine exposure to estrogen is associated with lower birth weight and higher BMI of the child and, furthermore, BMI is associated with pubertal development. ${ }^{39-41}$ However, we found no evidence of mediation after additional adjustment for birth weight and BMI of the children at seven years.

An important limitation is the potential misclassification of the exposure. First, nausea is not a perfect measure of intrauterine estrogen exposure. ${ }^{18}$ Second, nausea is a rather subjective perception which also may introduce misclassification in the reporting and this potential misclassification may have resulted in bias towards the null, and underestimation of the true association. A direct measure of maternal estrogen serum levels would have improved the study. Information on stages of pubertal development was also self-reported and may likewise be misclassified. However, an earlier validation study within the Puberty Cohort found moderate agreement between self-reported and clinical examination of pubertal milestones. ${ }^{42}$ Still, the risk of non-differential misclassification of our exposure outcome could potentially explain the lack of association. Some children in this study had already reached the earliest pubertal milestones before returning the first questionnaire. The earliest pubertal milestones were left censored for these children which may bias the estimates if the assumption of the normal distribution was violated. Reassuringly, the model diagnostics found that our data were reasonably compatible with the normal distribution. Further, age at attaining pubertal milestones has been shown to follow normality in healthy populations ${ }^{3}$ such as the Puberty Cohort. Finally, the finer categorization of our exposure information into 12 exposure levels based on the duration and severity of nausea and co-existence of vomiting could also introduce misclassification.

Another limitation was the risk of selection bias due to non-participation in the Puberty Cohort. However, participation was not associated with nausea in our study, a recent validation study found no association between a surrogate marker of pubertal timing and participation in the Puberty Cohort ${ }^{43}$ and we used selection weights. Hence, the risk of selection bias is probably minimal.

Severe nausea and vomiting during pregnancy may be treated pharmacologically. We cannot rule out that these treatments could confound the association between nausea during pregnancy and the pubertal development through pharmacological pathways. We were, however, able to adjust for potential confounders identified using a directed acyclic graph but we cannot rule out that residual confounding or confounding from unmeasured factors has affected the results.

The generalizability of our study is to some extent limited since the study population mainly consisted of Caucasians. Furthermore, the pregnancy care and the programs for pregnant women might differ between countries including in guidelines and treatments. However, the handling and treatment of nausea and vomiting during pregnancy is probably universal and therefore, the results of the study can to some extent be generalized to other populations.

\section{Conclusions}

In conclusion, we did not observe an association between nausea in the first trimester of pregnancy and altered mean age for pubertal milestones in sons and daughters. Neither did we observe any indications that duration or severity of nausea affect timing of puberty. In contrast, if maternal nausea acts as a surrogate marker of prenatal estrogen exposure, the present study does not support a programming role of pubertal timing by prenatal estrogen exposure in either boys or girls.

\section{Abbreviations}

BMI, body mass index; DNBC, Danish National Birth Cohort; hCG, human choriogonadotropin; HPG, hypothalamic-pituitary-gonadal. 


\section{Data Sharing Statement}

The data support the findings of this study available from the Danish National Birth Cohort, but restrictions apply to the availability of these data, which were used under license for the current study, and so are not publicly available. Data are, however, available from the authors upon reasonable request and with permission of the Danish National Birth Cohort.

\section{Ethics Approval and Informed Consent}

The Committee for Biomedical Research Ethics in Denmark approved the collection of data in the DNBC ((KF)01-471/ 94). A written informed consent was obtained from the mother upon recruitment including permission to follow-up until the child turned 18 years of age. The present study was approved by The Danish Data Protection Agency (2012-410379 and 2015-57-0002) and the Steering Committee of the DNBC (2012-04, 2015-47 and 2018-31).

\section{Consent for Publication}

A written informed consent was obtained from the mother upon recruitment including permission to follow-up until the child turned 18 years of age. No individual-level data are presented.

\section{Acknowledgments}

The Danish National Birth Cohort was established with a significant grant from the Danish National Research Foundation. Additional support was obtained from the Danish Regional Committees; the Pharmacy Foundation; the Egmont Foundation; the March of Dimes Birth Defects Foundation; the Health Foundation; and other minor grants. The DNBC Biobank has been supported by the Novo Nordisk Foundation; and the Lundbeck Foundation. Follow-up of mothers and children has been supported by the Danish Medical Research Council (SSVF 0646, 271-08-0839/06-066023, O602-01042B, 060202738B); the Lundbeck Foundation (195/04, R100A9193); The Innovation Fund Denmark (0603-00294B (09-067124)); the Nordea Foundation (02-2013-2014); Aarhus Ideas (AU R9-A959-13-S804); University of Copenhagen Strategic Grant (IFSV 2012); and the Danish Council for Independent Research (DFF - 418300594 and DFF - 4183-00152).

\section{Author Contributions}

All authors contributed to data analysis, drafting or revising the article, have agreed on the journal to which the article will be submitted, gave final approval of the version to be published, and agree to be accountable for all aspects of the work.

\section{Funding}

Financial and material support: This work was supported by the Danish Council for Independent Research (DFF 4183-00152), the Danish Medical Research Council (O602-01042B), the Faculty of Health at Aarhus University and the Aarhus University Research Fund (AU R9-A959-13-S804). The funders had no role in the design of the study; collection, analysis, and interpretation of data; and in writing the manuscript.

\section{Disclosure}

The authors declare that they have no competing interests.

\section{References}

1. Day FR, Elks CE, Murray A, Ong KK, Perry JR. Puberty timing associated with diabetes, cardiovascular disease and also diverse health outcomes in men and women: the UK Biobank Study. Sci Rep. 2015;5(1):11208. doi:10.1038/srep11208

2. Ong KK, Ahmed ML, Dunger DB. Lessons from large population studies on timing and tempo of puberty (secular trends and relation to body size): the European trend. Mol Cell Endocrinol. 2006;254255:8-12. doi:10.1016/j.mce.2006.04.018

3. Sorensen K, Mouritsen A, Aksglaede L, Hagen CP, Mogensen SS, Juul A. Recent secular trends in pubertal timing: implications for evaluation and diagnosis of precocious puberty. Horm Res Paediatr. 2012;77(3):137-145. doi:10.1159/000336325

4. Aksglaede L, Sorensen K, Petersen JH, Skakkebaek NE, Juul A. Recent decline in age at breast development: the Copenhagen Puberty Study. Pediatrics. 2009;123(5):e932-939. doi:10.1542/peds. 2008-2491

5. Golub MS, Collman GW, Foster PM, et al. Public health implications of altered puberty timing. Pediatrics. 2008;121(Suppl 3):S218-230. doi:10.1542/peds.2007-1813G

6. Kuiri-Hanninen T, Sankilampi U, Dunkel L. Activation of the hypothalamic-pituitary-gonadal axis in infancy: minipuberty. Horm Res Paediatr. 2014;82(2):73-80. doi:10.1159/000362414

7. Peper JS, Brouwer RM, van Leeuwen M, et al. HPG-axis hormones during puberty: a study on the association with hypothalamic and pituitary volumes. Psychoneuroendocrinology. 2010;35(1):133-140. doi:10.1016/j.psyneuen.2009.05.025

8. Lagiou P, Tamimi R, Mucci LA, Trichopoulos D, Adami HO, Hsieh CC. Nausea and vomiting in pregnancy in relation to prolactin, estrogens, and progesterone: a prospective study. Obstet Gynecol. 2003;101(4):639-644.

9. Verberg MF, Gillott DJ, Al-Fardan N, Grudzinskas JG. Hyperemesis gravidarum, a literature review. Hum Reprod Update. 2005;11 (5):527-539. doi:10.1093/humupd/dmi021

10. Broussard CN, Richter JE. Nausea and vomiting of pregnancy. Gastroenterol Clin North Am. 1998;27(1):123-151. doi:10.1016/ S0889-8553(05)70350-2 
11. Storgaard L, Bonde JP, Olsen J. Male reproductive disorders in humans and prenatal indicators of estrogen exposure. A review of published epidemiological studies. Reprod Toxicol. 2006;21(1):4-15. doi:10.1016/j.reprotox.2005.05.006

12. Masson GM, Anthony F, Chau E. Serum chorionic gonadotrophin (hCG), schwangerschaftsprotein 1 (SP1), progesterone and oestradiol levels in patients with nausea and vomiting in early pregnancy. $\mathrm{Br}$ J Obstet Gynaecol. 1985;92(3):211-215. doi:10.1111/j.1471-0528.19 85.tb01084.x

13. Furneaux EC, Langley-Evans AJ, Langley-Evans SC. Nausea and vomiting of pregnancy: endocrine basis and contribution to pregnancy outcome. Obstet Gynecol Surv. 2001;56(12):775-782. doi:10. 1097/00006254-200112000-00004

14. Christenson LK, Devoto L. Cholesterol transport and steroidogenesis by the corpus luteum. Reprod Biol Endocrinol. 2003;1(1):90. doi:10.1186/1477-7827-1-90

15. Franssen D, Ioannou YS, Alvarez-real A, et al. Pubertal timing after neonatal diethylstilbestrol exposure in female rats: neuroendocrine vs peripheral effects and additive role of prenatal food restriction. Reprod Toxicol. 2014;44:63-72. doi:10.1016/j.reprotox.2013.10.006

16. Pepe GJ, Lynch TJ, Albrecht ED. Regulation of baboon fetal ovarian development by placental estrogen: onset of puberty is delayed in offspring deprived of estrogen in utero. Biol Reprod. 2013;89(6):132. doi:10.1095/biolreprod.112.107318

17. Den Hond E, Schoeters G. Endocrine disrupters and human puberty. Int J Androl. 2006;29(1):264-271. discussion 286-290. doi:10.1111/ j.1365-2605.2005.00561.x

18. Body C, Christie JA. Gastrointestinal diseases in pregnancy: nausea, vomiting, hyperemesis gravidarum, gastroesophageal reflux disease, constipation, and diarrhea. Gastroenterol Clin North Am. 2016;45 (2):267-283. doi:10.1016/j.gtc.2016.02.005

19. Tsakiridis I, Mamopoulos A, Athanasiadis A, Dagklis T. The management of nausea and vomiting of pregnancy: synthesis of national guidelines. Obstet Gynecol Surv. 2019;74(3):161-169. doi:10.1097/ OGX.0000000000000654

20. Shehmar M, MacLean M, Nelson-Piercy C, Gadsby R, O’Hara M. The Management of Nausea and Vomiting of Pregnancy and Hyperemesis Gravidarum. Green-Top Guideline No.69; 2016.

21. Campbell K, Rowe H, Azzam H, Lane CA. The management of nausea and vomiting of pregnancy. $J$ Obstet Gynaecol Can. 2016;38(12):1127-1137.

22. Committee on Practice Bulletins-Obstetric. ACOG Practice bulletin no. 189: nausea and vomiting of pregnancy. Obstet Gynecol. 2018;131(1):e15-e30. doi:10.1097/AOG.0000000000002456

23. Malcolm KD, Jackson LM, Bergeon C, Lee TM, Padmanabhan V, Foster DL. Long-term exposure of female sheep to physiologic concentrations of estradiol: effects on the onset and maintenance of reproductive function, pregnancy, and social development in female offspring. Biol Reprod. 2006;75(6):844-852. doi:10.1095/biolreprod.106.053264

24. Hu J, Du G, Zhang W, et al. Short-term neonatal/prepubertal exposure of dibutyl phthalate (DBP) advanced pubertal timing and affected hypothalamic kisspeptin/GPR54 expression differently in female rats. Toxicology. 2013;314(1):65-75. doi:10.1016/j.tox.2013.09.007

25. The DNBC Puberty Follow-up [homepage on the Internet]. Copenhagen: the Danish National Birth Cohort; 2021. Available from: $\quad$ https://www.dnbc.dk/data-available/puberty-follow-up. Accessed September 22, 2021.https://www.dnbc.dk/data-available/ puberty-follow-up

26. Brix N, Ernst A, Lauridsen LLB, et al. Maternal smoking during pregnancy and timing of puberty in sons and daughters: a Population-Based Cohort Study. Am J Epidemiol. 2019;188 (1):47-56. doi:10.1093/aje/kwy206
27. Bliddal M, Broe A, Pottegard A, Olsen J, Langhoff-Roos J. The Danish medical birth register. Eur J Epidemiol. 2018;33(1):27-36. doi:10.1007/s10654-018-0356-1

28. The International Standard Classifications of Occupations [homepage on the Internet]. Switzerland: Department of Statistics; 2021. Available from: https://www.ilo.org/public/english/bureau/stat/isco/. Accessed September 22, 2021.

29. Wellner JA, Zhan Y. A hybrid algorithm for computation of the nonparametric maximum likelihood estimator from censored data. $J$ Am Stat Assoc. 1997;92(439):945-959. doi:10.1080/01621459. 1997.10474049

30. Huber J. The behavior of maximum likelihood estimates under nonstandard conditions. Econometrica. 1967;1:221-233.

31. White H. A heteroskedasticity-consistent covariance matrix estimator and a direct test for heteroskedasticity. Econometrica. 1980;48 (4):817-838. doi:10.2307/1912934

32. Hernan MA, Hernandez-Diaz S, Robins JM. A structural approach to selection bias. Epidemiology. 2004;15(5):615-625. doi:10.1097/01. ede.0000135174.63482.43

33. Steppan M, Whitehead R, McEachran J, Currie C. Family composition and age at menarche: findings from the international health behaviour in School-Aged Children Study. Reprod Health. 2019;16 (1):176. doi:10.1186/s12978-019-0822-6

34. Johnson W, Choh AC, Curran JE, et al. Genetic risk for earlier menarche also influences peripubertal body mass index. Am J Phys Anthropol. 2013;150(1):10-20. doi:10.1002/ajpa.22121

35. Roseboom T, de Rooij S, Painter R. The Dutch famine and its long-term consequences for adult health. Early Hum Dev. 2006;82 (8):485-491. doi:10.1016/j.earlhumdev.2006.07.001

36. Poeran-Bahadoer S, Jaddoe VWV, Gishti O, et al. Maternal vomiting during early pregnancy and cardiovascular risk factors at school age: the Generation R Study. J Dev Orig Health Dis. 2020;11(2):118-126. doi:10.1017/S2040174419000114

37. Grooten IJ, Painter RC, Pontesilli M, et al. Weight loss in pregnancy and cardiometabolic profile in childhood: findings from a longitudinal birth cohort. BJOG. 2015;122(12):1664-1673. doi:10.1111/1471-05 28.13042

38. Ahmed ML, Ong KK, Dunger DB. Childhood obesity and the timing of puberty. Trends Endocrinol Metab. 2009;20(5):237-242. doi:10. 1016/j.tem.2009.02.004

39. Kaplowitz PB. Link between body fat and the timing of puberty. Pediatrics. 2008;121(Suppl 3):S208-217. doi:10.1542/peds.2007$1813 \mathrm{~F}$

40. Wang Y. Is obesity associated with early sexual maturation? A comparison of the association in American boys versus girls. Pediatrics. 2002;110(5):903-910. doi:10.1542/peds.110.5.903

41. Frisch RE, Revelle R. Height and weight at menarche and a hypothesis of menarche. Arch Dis Child. 1971;46(249):695-701. doi:10.1136/adc.46.249.695

42. Ernst A, Lauridsen LLB, Brix N, et al. Parental time to pregnancy, medically assisted reproduction and pubertal development in boys and girls. Hum Reprod. 2019;34(4):724-732. doi:10.1093/humrep/ dez008

43. Brix N, Ernst A, Lauridsen LLB, et al. Risk of selection bias due to non-participation in a cohort study on pubertal timing. Paediatr Perinat Epidemiol. 2020;34(6):668-677. doi:10.1111/ppe.12679 


\section{Publish your work in this journal}

Clinical Epidemiology is an international, peer-reviewed, open access, online journal focusing on disease and drug epidemiology, identification of risk factors and screening procedures to develop optimal preventative initiatives and programs. Specific topics include: diagnosis, prognosis, treatment, screening, prevention, risk factor modification,

Submit your manuscript here: https://www.dovepress.com/clinical-epidemiology-journal systematic reviews, risk \& safety of medical interventions, epidemiology \& biostatistical methods, and evaluation of guidelines, translational medicine, health policies \& economic evaluations. The manuscript management system is completely online and includes a very quick and fair peer-review system, which is all easy to use. 